\title{
LAS ACTITUDES CIENTIFICAS DE LOS FUTUROS MAESTROS EN RELACION CON SUS CONOCIMIENTOS
}

\author{
ESCUDERO ESCORZA, T. Y LACASTA ZABALZA, E. \\ Instituto de Ciencias de la Educación (Universidad de Zaragoza) \\ E.U. de Formación del Profesorado de EGB de Navarra (Universidad de Zaragoza).
}

\section{SUMMARY}

This work attemps to analyze in depth scientific attitudes and their relationship with scientific knowledge in Physics, in students of Science in a Teacher Training School. The influence of sex and the differences with a sample of American students are considered in detail.

\section{INTRODUCCION}

En las reuniones, seminarios y congresos sobre formación del profesorado que se celebran en muestro pais, siempre llegamos a la conclusión de que es necesario reforzar la formación y actitudes didácticas, en con. sonancia con una sólida preparación disciplinaria. Estas conclusiones, con carácter general, son aplicadas con los matices convenientes a la formación de futu. ros profesores de ciencia básica en nuestras E.U. de Formación del Profesorado, donde nos preocupa que se consiga la debida formación científica y que los conocimientos generales de pedagogia y didáctica se canalicen hacia una didáctica especial entroncada con el nivel y con las disciplinas científicas.

Lo que quizás se olvida con más frecuencia y no solamente en nuestro pais, es el análisis de la necesidad de fomentar en el profesorado las actitudes científicas requeridas para llevar a cabo su labor de manera rigurosa. En el caso del profesorado de ciencias, la adquisición de actitudes científicas es si cabe, todavía más crí. tica y relevante.

Un maestro que no posea en alto grado objetividad, disposición al cambio de opinión, mente crítica, etc., difícilmente podrá iniciar a sus alumnos en los caminos de la ciencia correctamente entendida y difícilmente podrá actuar como un científico en su propia clase. laboratorio, como nos sugiere Stenhouse (1976), experimentando en la mejora de su propia labor docente.

La realidad de la formación en ciencias que se imparte en nuestras E.U. del Profesorado de EGB, es que se procura que el alumno conozca los fundamentos de la ciencia y, en el mejor de los casos, que esté capacitado para llevar a cabo actividades cientificas prácticas, pe ro se olvida a menudo de analizar y comprobar si esos futuros maestros tienen ese talante científico y las actividades que son necesarias para llevar a sus aulas la experimentación, el análisis, el contraste de ideas, etc. Sin actitudes científicas es difícil que el maestro las pueda promover en sus alumnos y dudoso que avance de manera eficaz en sus intentos de innovación educativa.

Las actitudes cientificas tampoco han recibido excesivo impulso de investigación si nos remitimos a las publicaciones existentes sobre el tema. Las revistas especializadas en didáctica de las ciencias ofrecen muy frecuentemente trabajos sobre las actitudes ante la ciencia, pero muy esporádicamente sobre las actitudes científicas. Las dificultades de conceptualización y de medición pueden ser la razón de este escaso desarrollo.

A pesar de las dificultades, han sido varios los instrumentos de medición de actitudes científicas que se han elaborado en las últimas décadas. Algunos de los ejemplos más representativos sería el elaborado por Noll (1933 y 1935), Cooley (1961) y el TOSA (Test on Scientific Attitudes) de Kozlow y Nay (1976).

Todos estos instrumentos citados y otros varios, tienen evidentemente limitaciones métricas de cierta importancia; aunque es notable el avance progresivo con el paso de los antos, el desarrollo instrumental ha sido claramente acumulativo. Los problemas de precisión en la conceptualización son visibles en muchos casos, asi como las dificultades para distinguir claramente los componentes de las actitudes.

A partir de un inventario de atributos afectivos del científico elaborado por Nay y Crocker (1970) y de los plan- 
teamientos de Rokeach (1968) en cuanto a las dimensiones de las actitudes, Kozlow y Nay (1976) parten de tres componentes de las actitudes cientificas: cognoscitivo, de propósito y de acción. El componente cog. noscitivo representa la comprensión por parte del estudiante de la manera en que las actitudes se manifiestan en la conducta profesional del científico. El componente de propósito representa la tendencia del estudiante para mostrar acuerdo o desacuerdo con conductas que definen una actitud. El componente de acción representa la medida en que el estudiante demuestra en la clase, de ciencias en este caso, las conductas definitorias de una actitud. El TOSA de Kozlow y Nay consta de cuarenta «items", los veinte primeros recogen el componente cognoscitivo (CCS-Cognitive Component Subtest) y los veinte últimos «items" el componente de proposito (ICS - Intent Component Subtest). El componente de acción requiere obviamente el apoyo de la observación directa.

Lacasta (1982) tradujo y adaptó el TOSA al castellano, guardando la máxima fidelidad a la fuente original. La terminologia para denominar el test que usó, y que es la empleada en este trabajo, fue la de Test so. bre las Actitudes Cientificas (TAC), Subtest del Componente Cognoscitivo (SCC) y Subtest del Componente de Propósito (SCP). En el presente artículo vamos a recoger los principales resultados de un estudio llevado a cabo con el TAC en la E.U. del Profesorado de EGB de Navarra, en el que se analizan sus relaciones con un test de conocimientos de física y las diferencias que aparecen en razón del sexo.

\section{OBJETIVOS DEL ESTUDIO}

El propósito más global del trabajo es el de contribuir a una mejor formación del profesorado de Ciencias de EGB, analizando Ios conocimientos y las actitudes cientificas de los alumnos de magisterio e intentando bus. car vías para solucionar problemas que se detectan. $\mathrm{El}$ contenido de este artículo no recoge sino parte de unos trabajos que están previstos con mayor alcance y continuidad.

Los objetivos específicos del presente estudio son los siguientes: a) conocer las actividades y conocimientos cientificos de los alumnos de magisterio de la especia. lidad de ciencias, b) estudiar la relación entre la madurez académica en Física y las actitudes cientfficas en dichos alumnos, c) replicar el estudio de Kozlow y Nay en otro contexto y contrastar los resultados, con vistas a proporcionar nuevas evidencias empíricas para la validación del instrumento de medición de actitudes y d) analizar la influencia del sexo en materia de actitudes científicas.

Dacias las características del estudio no partíamos de hipótesis previas, aunque contábamos con un elemento básico de contraste como el de los resultados de Kozlow y Nay.

\section{INSTRUMENTOS DE MEDICION}

Las actitudes científicas fueron medidas con el TAC (Test sobre las Actitudes Cientificas) según la version de Lacasta (1982) traducida del TOSA. Como ya hemos apuntado, el TAC consta de cuarenta «items" y está dividido en dos subtests. El Subtest del Componente Cognoscitivo (SCC) lo constituyen los items del 1 al 20, en los que el estudiante debe seleccionar una respuesta entre cuatro actuaciones referentes a una situación determinada. El Subtest del Componente de Propósito (SCP) lo constituyen los items del 21 al 40 , en los que el estudiante debe elegir entre cuatro iniciativas la que mejor describa su intención ante una situación dada.

El conjunto del test es ofrecido en las fuentes antes mencionadas y por razones de espacio no parece oportuno reproducirlo, sin embargo, a modo de ejemplo, ofrecemos dos items, uno del SCC (item 17) y otro del SCP (item 24).

Item 17. Un científico demuestra que tiene una mente abierta cuando:

A. Discute sus ideas con otros científicos

B. Valora y considera ideas que no están de acuerdo con sus teorías.

C. Está de acuerdo con las ideas presentadas por otros científicos.

D. Demanda a los demás científicos pruebas experimentales para apoyar sus argumentos.

Item 24. "La luz se propaga como un haz de partículas".

«La luz se propaga como una onda»

Si encuentras estas dos afirmaciones en dos libros diferentes, ¿cuál de las siguientes acciones te parece la mejor?

A. Preguntar a tu profesor cuál de las dos afirmaciones hay que aceptar

B. Consultar ese tema en otros libros

C. Asumir que los científicos no están seguros de cómo se provaga la luz

D. Aceptar lo que afirma el libro más reciente.

Los análisis racionales y factoriales llevados a cabo por Kozlow y Nay presentan una división estructural del test en seis actitudes cientfficas distintas, que nosotros hemos analizado también de forma separada. Las seis actitudes son las siguientes: Mente critica (MC), Juicio Controlado o Selectivo (JC), Respeto por la Evidencia (RE), Honestidad $(\mathrm{H})$, Objetividad (O) y Disposición al Cambio de Opinión (DC). Estas actitudes están definidas por diferentes «items» de ambos subtests, con «items» o rasgos comunes en algún caso. Así por ejemplo, el rasgo de «informar de sus observaciones incluso cuando contradicen sus hipotesis", contribuye a definir las actitudes de Honestidad y Objetividad. 
El otro instrumento de recogida de información utilizado fue un Test de Conocimientos de Física (TCF) elaborado por Lacasta (1982), a partir de los contenidos de Física en EGB y BUP y atendiendo a distintas destrezas de razonamiento, aplicación, análisis, etc., pe* ro reduciendo el aparato metemático y el requisito de información aprendida, que con frecuencia es aportada en la propia formulación de las preguntas. El formato del TCF es el mismo que el del TAC: elección múltiple con cuatro alternativas.

Tras una aplicación piloto a un grupo de alumnos de COU y el correspondiente análisis de items, revisión de distractores, etc., el TCF definitivo quedó reducido a treinta y una cuestiones.

\section{SUJETOS DEL ESTUDIO}

Concluida la fase de preparación y corrección definitiva de los tests de actitudes y conocimientos, se aplicaron a todos los alumnos de los cursos primero y segundo de la especialidad de Ciencias de la E.U. de Formación del Profesorado de EGB de Navarra (Univer. sidad de Zaragoza). La aplicación se hizo en la primera fase del curso académico en sesiones dentro del horario lectivo como mejor medio para disminuir potenciales sesgos de selección.

En un principio, el número total de alumnos que correspondieron a los test fue de 141 , pero hubo que desechar cinco sujetos que habian aportado información incompleta. En definitiva, la muestra total fue de $\mathrm{N}$ $=136$ alumnos de los que 69 cursaban segundo curso (21 varones, y 46 mujeres).

\section{ANALISIS DE DATOS}

El tratamiento estadistico y el análisis de datos fue llevado a cabo a través de un programa de computador de la Biblioteca del "Teacher College Computer» de la Universidad de Winsconsin, adaptado al sistema del Centro de Cálculo de la Universidad de Zaragoza en un estudio de Escudero (1981) sobre niveles de madu. rez al final de la EGB. Este programa realiza un análisis de «items» muy completo y riguroso, indicando porcentajes de respuesta e índices de dificultad y discrimi nación en valores $Z$, de manera que los valores negativos indican poca dificultad y discriminación. Así mismo, el programa calcula para el conjunto del test y cada subtest, la media, la desviación típica y el coeficiente de consistencia interna de Kuder-Richardson ( $\mathrm{r}_{\mathrm{KR}}{ }^{20}$ ).

Los estudios de correlación fueron llevados a cabo con el programa SPSS 11 en su versión V3.0 de NIE y colaboradores (1975). El paquete SPSS 11 en sus distin. tas versiones es una adecuación de los programas SPSS al sistema PDP 11.

Las variables correlacionadas entre si fueron las pun" tuaciones en los Test de Conocimiento de Física (TCF) y Actitudes Científicas (TAC), en los subtests de Componente Cognoscitivo (SCC) y Componente de Propo- sito (SCP) y en las seis actitudes previamente señaladas: Mente Critica (MC), Juicio Controlado (J.C.), Respeto por la Evidencia (RE), Honestidad (H), Objetividad (O) y Disposición al Cambio de Opinión (DC).

Todos estos análisis los llevamos a cabo para la muestra total y para las distintas submuestras según los criterios de estratificación, esto es, sexo y curso.

\section{CONOCIMIENTO EN FISICA}

En la Tabla 1 aparecen los valores de la media, desviación típica y $\mathrm{r}_{\mathrm{kR}^{20}}$ para el Test de Conocimientos de Física (TCF) en la muestra total y cada una de las submuestras.

\begin{tabular}{|l|c|c|c|}
\hline \multicolumn{4}{|c|}{ Test de Conocimiento de Física } \\
\hline Muestra & Media & $\begin{array}{c}\text { Desviación } \\
\text { Típica }\end{array}$ & $\mathrm{r}_{\mathrm{KR}^{20}}$ \\
\hline Primero & 19,04 & 3,34 & 0,50 \\
\hline Segundo & 18,48 & 3,52 & 0,52 \\
\hline Varones & 19,61 & 3,86 & 0,63 \\
\hline Mujeres & 18,44 & 3,21 & 0,42 \\
\hline TOTAL & 18,77 & 3,43 & 0,50 \\
\hline
\end{tabular}

Tabla 1.

Resultados del Test de Conocimientos de Física (TCF)

Los resultados anteriores nos presentan una acusada igualdad entre los distintos estratos y cierta homogeneidad en las distribuciones. Los varones ofrecen va* lores algo más altos en media, dispersión y consistencia interna. El TCF of rece una dificultad media, poca dispersión y una consistencia interna razonable en un test que abordaba un espectro amplio de contenidos. Téngase presente que en primer curso no existe la asignatura de Física.

Como observaciones más destacables hay que señalar el alto número de omisiones en preguntas de Optica y, en menor medida, Electromagnetismo, lo que parece indicar que son partes poco tratadas en los programas de BUP, así como el predominio de la intuición sobre la asimilación y aplicación de los principios de la Física clásica, incluso en los alumnos de alto rendimiento como lo demostraban ciertos valores del índice de dis" criminación (Zd). También se observa una media de rendimiento ligeramente más alta en los varones que en las mujeres.

A pesar de que en el mismo TCF aparecía el enunciado de los tres principios de la dinámica, la mayoría de los alumnos elegían distractores apoyados en la idea de que «si no hay fuerza, no hay movimiento». Ante este y otros datos similares, cabe preguntarse si estos 
alumnos que en su mayoria no han incorporado a su razonamiento la Física de Galileo, podrán adquirir en menos de dos años una concepción actualizada de la Ciencia y, lo que es más importante, transmitirla correctamente a sus futuros alumnos.

Ante esta observacion hay que decir que el tema de los errores conceptuales es de interés para muchos investigadores de la enseñanza de las ciencias como denuestra Carrascosa en la selección bibliográfica que ofrece en el $n^{\circ} 1$ (vol. 1) de esta misma revista. En nuestro propio país han publicado trabajos sobre este problema, el propio Carrascosa, Gil, Furió, González, Ortiz y otros.

\section{ACTITUDES CIENTIFICAS}

Los resultados en el Test sobre las Actitudes Cientfficas (TAC) y en los dos subtests (SCC y SCP) en muestros alumnos, los comparamos con los que ofrecen Kozlow y Nay (1975) para una muestra de 307 alumnos americanos de Fisica y Quimica del $11^{\circ}$ grado (equivalente aproximadamente al COU de Ciencias), no solamente para ofrecer nuevos elementos para la validación del instrumento de evaluación, sino también para tener un elemento de contraste de la situación de nuestros alumnos.

En la Tabla II reproducimos los valores obtenidos para la media, desviación típica y coeficiente de fiabilidad para el test de actitudes y los dos subtests en todas nuestras submuestras y en la Kozlow y Nay. Hemos transformado todos los resultados a escala $(0-100)$ porque es así como ofrecen los resultados Kozlow y Nay.

De la tabla anterior podemos destacar algunas obser. vaciones como que nuestros alumnos, como podía esperarse por su mayor nivel académico, tienen unos promedios algo más altos que los de la muestra de Kozlow y Nay y, además, sus respuestas son algo más homogéneas. Sin embargo, el test es mucho menos consistente con nuestros alumnos de lo que indican Kozlow y Nay, sobre todo por la escasísima consistencia del subtest de propósito. Con el subtest cognoscitivo nos sucede algo parecido a lo que reportan Kozlow y Nay, pero con el subtest de propósito disminuyen muchísimo los valores del findice de consistencia interna. Como ya apuntaban estos autores, el TAC y sobre todo el SCP, contiene preguntas que no están muy relacionadas entre si y que pueden ser vistas de manera diferente según las características de la muestra a la que se aplica.

Al contrario de lo que ocurria con los conocimientos, los alumnos de segundo presentan unas medias de actitudes cientificas ligeramente superiores que los primeros, sin embargo, el SCP tiene mucha menor consistencia con los de segundo que con los de primero.

Los varones presentan actitudes promedio más altas que las mujeres, especialmente en el componente cognoscitivo.

Al analizar la elección de distractores y los resultados de los distintos «items», parece desprenderse que nuestros alumnos están menos influenciados que los de Kozlow y Nay por ideas religiosas y extra-cientfficas, sin embargo, otras respuestas inducen a pensar que al mismo tiempo están menos familiarizados con el papel que juegan las pruebas empíricas en una investigación, mostrando un menor respeto por la evidencia que los alumnos de Kozlow y Nay.

\begin{tabular}{|c|c|c|c|c|c|c|c|c|c|}
\hline Muestra & 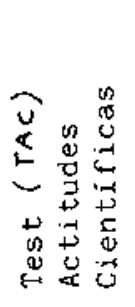 & 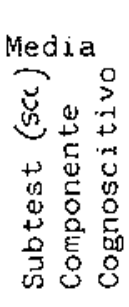 & 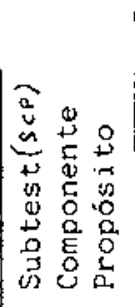 & 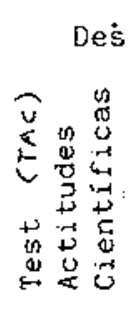 & 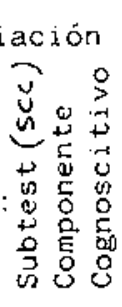 & 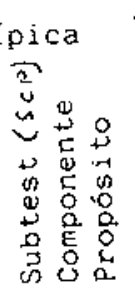 & 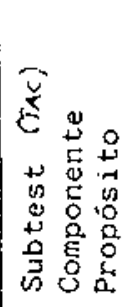 & 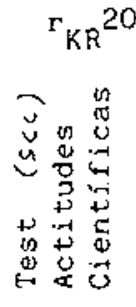 & 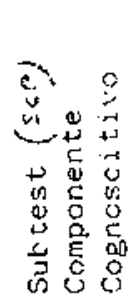 \\
\hline Primero & 52,8 & 53,6 & 55,1 & 8,3 & 11,8 & 10,8 & 0,30 & 0,30 & 0,20 \\
\hline Segundo & 55,9 & 56,7 & 55,2 & 8,7 & 11,9 & 9,3 & 0,40 & 0,39 & $-0,1 \mathrm{~J}$ \\
\hline Varones & 58,2 & 59,5 & 57,0 & 8,0 & 11,8 & 9,6 & 0,27 & 0,34 & $-0,03$ \\
\hline Mujeres & 52,8 & 51,3 & 54,4 & 8,4 & 11,6 & 10,2 & 0,33 & 0.31 & 0,08 \\
\hline Total & 54,3 & 53,6 & 55,1 & 8,6 & 12,2 & 10,0 & 0,36 & 0,38 & $0,0.5$ \\
\hline $\begin{array}{l}\text { Ko\%low } \\
\text { y May }\end{array}$ & 52,4 & 52,8 & 52,2 & 10,3 & 13,9 & 12.5 & 0,55 & 0,45 & 0.39 \\
\hline
\end{tabular}

Tabla 2. Resultado en el test de actitudes 


\section{RELACIONES DEL CONOCIMIENTO Y AC- TITUDES}

El estudio de las actitudes gana interés e importancia si se relaciona con el conocimiento disciplinario y con esta perspectiva planteamos nuestro trabajo. El estudio de Kozlow y Nay no recoge este aspecto y se limita a correlacionar el TOSA con un Test de lectura, quizás porque su principal interés era la construcción del test y no consideraron la telación con el conocimiento como algo relevante en dicho proceso.

Nosotros hemos correlacionado entre sí el TCF, el $\mathrm{TAC}$, los dos subtests y las puntuaciones en las seis actitudes obteniendo una matriz de correlaciones $10 \times 10$ para cada muestra. En la tabla 3 ofrecemos los coeficientes de correlación entre el Test de Conocimientos de Física (TCF) y todas las medidas de actitudes para los distintos estratos.

A nuestro entender, el más importante resultado de la Tabla II es que alumnos y alumnas ofrecen unas relaciones totalmente diferentes, de forma que los resultados relativos a las otras muestras tienen significación confusa por la mezcla de varones y mujeres. En estas no existe ninguna relación significativa entre el conocimiento y las actitudes, mientras que en aquellos la relación es sistemática, positiva y en algứn caso considerable. La disposición al Cambio de Opinión (DC), el Test sobre las Actitudes Científicas (TAC) en su conjunto y la Honestidad $(\mathrm{H})$ son las variables que pre- senta el más alto nivel de significación en su relación con el Test de Conocimientos de Ffsica.

Esta diferencia entre varones y mujeres es algo que se recoge en un buen número de trabajos sobre didáctica de las Ciencias, como es bien conocido por los estudiosos del tema y se refleja en los textos y publicaciones especializadas. Basta recordar las diferencias entre sexos observadas en el conocido estudio de Comber y Keeves (1973).

El Subtest de Componente Cognoscitivo tiene una relación muy débil con el de Propósito $(0,11$ para varones y 0,18 para mujeres, 0,23 para Kozlow y Nay). Por otra parte, las distintas parejas de actitudes tienen correlaciones moderadas o bajas, aunque sean significativas, en cerca de la mitad de las parejas que se pueden formar, si bien es cierto que en muchos casos existe algún «item» común. Si se controlan estas circunstancias, las correlaciones que parecen tener más significación son las de la actitud Mente Crítica (MC) con el Juicio Controlado (JC), Honestidad (H), Objetividad (O) y Disposición al Cambio de Opinión (DC) y las de la Honestidad (H) con el Juicio Controlado (JC) y la Disposición al Cambio de Opinión (DC). La actitud Mente Crítica (MC) es la más relacionada con el resto, mientras que el Respeto por la Evidencia (RE) aparece como totalmente independiente del resto, salvo en el caso del Juicio controlado (JC) con la que tiene tres «items» comunes.

\begin{tabular}{|c|c|c|c|c|c|c|c|c|c|}
\hline Muestra & 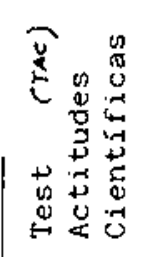 & 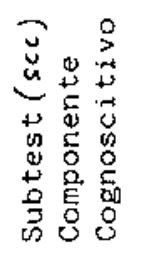 & 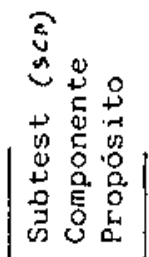 & 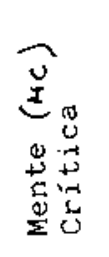 & 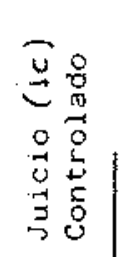 & 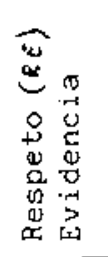 & 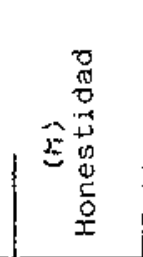 & $\begin{array}{r}0 \\
0 \\
0 \\
0.5 \\
0.0 \\
0.0 \\
0.0 \\
0 \\
0 \\
0\end{array}$ & 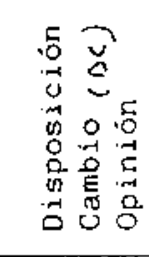 \\
\hline $\begin{array}{l}\text { Prinero } \\
(N=69)\end{array}$ & $2 A^{*}$ & 19 & 16 & 7 & 1.1 & 09 & 6 & 17 & 7 \\
\hline $\begin{array}{l}\text { Segundo } \\
(N=67)\end{array}$ & 19 & 20 & 11 & 13 & 14 & 16 & 12 & 13 & $28 * *$ \\
\hline $\begin{array}{l}\text { Varones } \\
(N=38)\end{array}$ & $48 * *$ & $35^{*}$ & $37^{*}$ & $27^{*}$ & $33^{*}$ & $28^{*}$ & $41 * *$ & 17 & $58^{* *}$ \\
\hline $\begin{array}{l}\text { Mujeres } \\
(N=98)\end{array}$ & 3 & 2 & 2 & -4 & -2 & -1 & -13 & 13 & -8 \\
\hline $\begin{array}{l}\text { TOTAL } \\
(N=136)\end{array}$ & $20^{*}$ & $16^{*}$ & 13 & 8 & 11. & 10 & 7 & $15^{*}$ & $15^{*}$ \\
\hline
\end{tabular}

\footnotetext{
- significativo al nivel $p \leqslant 0,05$

* significativo al nivel $p \leqslant 0,01$
}

Tabla 3. Correlaciones del Test de Conocimientos de Fisica con las Actitudes cientificas, (los indices de correlación están multiplicados por 100). 


\section{CONCLUSIONES Y COMENTARIOS}

Los resultados que acabamos de presentar y analizar nos confirman la idea de que el importante terreno de las actitudes científicas tiene mucho de inexplorado todavia, existiendo muchos problemas por resolver en cuanto a su definición y medición.

Si nos atenemos a los resultados analizados, nos resulta difícil pensar en un constructo único de actitud científica porque aparecen distintos rasgos con cierta independencia. Asimismo, el componente congnoscitivo y el de propósito no parecen tener relación como para pensar en su integración en un concepto único en un futuro cercano. En este mismo sentido inciden los resultados que presentan Kozlow y Nay para el componente de acción. Habrá que pensar en la necesidad de fomentar de manera independiente las distintas actitudes cientificas que se consideran relevantes en el proceso de formación de científicos y profesores de Ciencias.

En cuanto al conocimiento disciplinario hemos visto que tiene una relación positiva y sistemática con las distintas actitudes cientfficas en el caso de los varones, pero no en el de las mujeres. Para éstas, los planes de formación centrados únicamente en los conocimientos no aportarían nada a la promoción de las actitudes científicas; se requeririan acciones específicas en tal sentido. La ensellanza de conocimientos no fomenta por sí misma las actitudes cientificas en los futuros maestros; las refuerza moderadamente en los varones, pero no en las mujeres.

\section{REFERENCIAS BIBLIOGRAFICAS}

COMBER, L.C. y KEEVES, J.P., 1973, Science Education in Nineteen Countries (John Wiley Inc Sons: New York).

ESCUDERO ESCORZA, T,, 1981, Anólisis de perfiles de madurez en las áreas científicas, lingüistica y matemática al término de la $E G B$ (ICE: Zaragoza).

LACASTA ZABALZA, E., 1982, Conocimientos de Física en relación con la actitud entre la Ciencia, en alumnos de Escuela de Magisterio, (Memoria de Licenciatura: Zaragoza).

KLOPFER, L.E, y COOLEY, W.W., 1961,Tous: Test on Understanding Science, Form $W$. (Educational Testing Service: Princeton).

KOZLOW, M.J. y NAY, M.A., 1976, An approach to measuring scientific attitudes, Science Education, Vol. 60, pp. 147-172.
El Test sobre las Actitudes Cientificas (TAC) y sus distintos componentes es un instrumento potencialmente muy valioso e interesante pero que requiere sustanciales mejoras en su validez interna y externa. En este trabajo solamente se aportan algunos datos nuevos a los ya existentes previamente en el camino de tal mejora, pero quedan muchos puntos abiertos para nuevos aná- lisis. El tema de la diferencia entre sexos es un importante punto para futuras investigaciones.

La conclusión operativa más global, siempre desde la prudencia que requiere el moverse en un terreno con un desarrollo relativamente escaso, es que la promoción de actitudes cientificas en los futuros profesores de EGB (Especialidad de Ciencias), debe fomentarse independientemente de su preparación en conocimientos cientificos. Así mismo, este desarrollo actitudinal tendría que llevarse a cabo a partir de objetivos específicos para las distintas actitudes y no a partir de un objetivo general que pretenda englobar el concepto de actitud cientifica.

La consecución de un profesor de ciencias básicas que se encuentra cómodo en todos los dominios educativos requerirá, con toda seguridad, una atención a todos ellos, pero de manera coordinada y globalizada. Las clases de nuestras E.U. del Profesorado necesitan convertirse en laboratorios en los que se abordan temas disciplinarios e interdisciplinarios siguiendo el esquema y el proceso del quehacer científico, en el que el profesor y los futuros maestros tengan necesidad de poner constantemente a prueba su talante científico y de actuar científicamente.

NAY, M.S. y CROCKER, R.R., 1970, Science teaching and the affective attributes of scientists, Science Education, Vol., 54, pp. 59-67.

NIE, N.H. y otros, 1975, Stadistical package for the Social Sciences (2* edicion ) (McGraw-Hill: New York).

NOLL, V.H., 1933, The Habit of scientific thinking, Teacher College Records, Vol. 35, pp. 1-9.

NOLL, V.H., 1935, Measuring the scientific attitude, The Journal of Abnormal and Social Psychology, Vol. 30, pp. 145-154.

ROKEACH, M. , 1968, Beliefs, Attitudes and values (Jossey Bass Inc.: San Francisco).

STENHOUSE, L., 1976, An introduction to curriculum research and development. (Heinemann: Londres). 